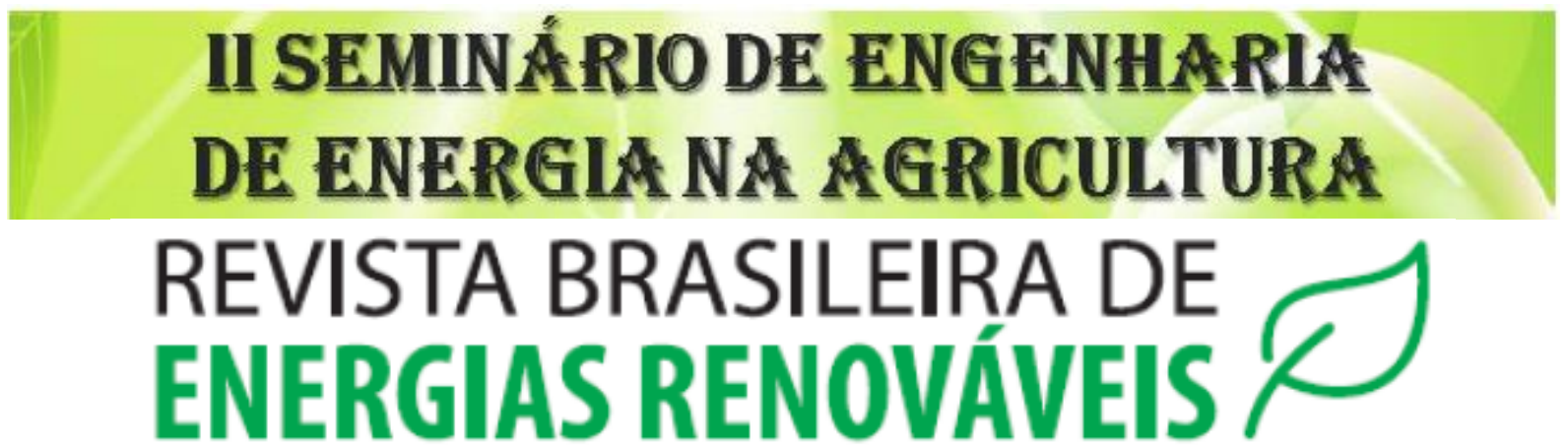

\title{
ANÁLISE DE SISTEMA HIDROCINÉTICO COMO CONVERSÃO DE ENERGIA PARA BOMBEAMENTO DE ÁGUA ${ }^{1}$
}

\author{
Camila Zeni Do Amaral ${ }^{2}$, Julio Cezar Girardi ${ }^{3}$, Soni Willian Haupenthal ${ }^{3}$
}

\footnotetext{
${ }^{1}$ Apresentado no $2^{\circ}$ Seminário de Engenharia de Energia na Agricultura: 30/11/2017UNIOESTE, Campus Cascavel.

${ }^{2}$ Universidade Tecnológica Federal do Paraná - UTFPR, Bacharelado em Engenharia Ambiental - Nível Graduação, Medianeira-PR. camilazamaral@gmail.com

${ }^{3}$ Universidade Estadual do Oeste do Paraná - UNIOESTE, PPGEA - Programa de Pós Graduação em Engenharia de Energia na Agricultura - Nível Mestrado, Cascavel-PR.
}

\section{RESUMO}

Como uma alternativa sustentável para a geração de energia elétrica, a energia hidrocinética é capaz de converter a energia cinética dos rios ou corrente marinhas em energia elétrica. A partir disso, este artigo tem como objetivo apresentar uma revisão de literatura, acerca da relação de informações práticas e conhecimento científico, a fim de maximizar os estudos acerca do tema apresentado e contribuir com informações sobre a utilização de energias renováveis aplicadas ao bombeamento de água, discorrendo os componentes do sistema hidrocinético de geração de energia, fatores de desempenho e vantagens aplicados ao bombeamento de agua. Assim, o sistema hidrocinético contribui para a eliminação do uso de combustíveis fósseis e soluções renováveis, dando acesso à energia elétrica em locais remotos.

Palavras-chave: bombeamento, energia renovável, sistema hidrocinético. 


\title{
HYDROKINETIC SYSTEM ANALYSIS AS ENERGY CONVERSION FOR WATER PUMPING.
}

\begin{abstract}
As a sustainable alternative for the generation of electric energy, hydrokinetic energy is able to convert the kinetic energy of rivers or marine currents into electric energy. From this, this article aims to present a literature review about the relationship of practical information and scientific knowledge, in order to maximize the studies about the presented theme and contribute with information on the use of renewable energies applied to water pumping, discussing the components of the hydrokinetic energy generation system, performance factors and advantages applied to water pumping. Thus, the hydrokinetic system contributes to the elimination of the use of fossil fuels and renewable solutions, giving access to electricity in remote places.
\end{abstract}

Keyword: pumping, renewable energy, hydrokinetic system.

\section{Introdução}

A adoção de energias alternativas tem sido amplamente buscada desde a década de 1970, quando as crises do petróleo levaram diversos países a procurar a segurança no fornecimento de energia e a redução da dependência da importação de combustíveis. Recentemente, as preocupações ambientais se tornaram o maior motor para a busca de alternativas mais limpas de produção de energia.

$\mathrm{Na}$ tentativa de mitigação desses problemas, as energias alternativas, estão ganhando espaço devido a sua sustentabilidade e baixo impacto ambiental durante operação. No Brasil, destacam-se a energia eólica, a solar, a biomassa e a hidroelétrica. A última delas abrange, comumente, as turbinas hidráulicas convencionais e as turbinas hidrocinéticas (FIGUEIREDO, 2013).

Para obtenção de energia elétrica de fontes renováveis é necessário um estudo inicial, no qual se verifica a viabilidade do potencial disponível. Os aspectos considerados para determinar a 
viabilidade do projeto, levam em conta os critérios técnicos, econômicos, sociais e ambientais. Mesmo que haja disponibilidade de uma fonte de energia e não havendo demanda, o projeto não alcançará os objetivos econômicos e sociais. A questão técnica envolve uma análise do potencial disponível e a verificação da melhor maneira de obtenção dessa energia, de forma que o aproveitamento tenha o rendimento esperado (WU et al, 2016).

A energia hidrocinética surgiu como uma alternativa sustentável para a geração de energia elétrica, a qual é capaz de converter a energia cinética dos rios ou correntes marinhas, em energia elétrica.

A instalação de turbinas hidrocinéticas pode ter um efeito significativo e complexo em padrões de fluxo, processos sedimentares e biológicos. De várias questões em termos de viabilidade tecnoeconômica, otimização de projeto, confiabilidade, colocação de turbina no canal, aplicação de aumento de duto, ancoragem e monitoramento ambiental são ainda precisava ser considerado antes da adoção generalizada desta tecnologia (LAGO, 2010).

\section{- Elementos do sistema hidrocinético de bombeamento de água (SEBA)}

Diversas tecnologias hidrocinéticas estão em desenvolvimento, principalmente relacionadas à aplicações em energia oceânica. O sistema HKEC consiste em cinco subsistemas HKT, gerador, estrutura de suporte, sistema de controle e sistema de transmissão (KUMAR et al., 2016).

Diversas tecnologias foram desenvolvidas para extrair energia hidrocinética, como sistemas de flutuação ou boia e dispositivo de coluna. Entre essas tecnologias, as turbinas hidrocinéticas são um dos mais utilizados. Essas turbinas possuem similaridade com as turbinas eólicas em termos de princípios físicos de operação, hardware elétrico e capacidade de velocidade variável para extração ideal de energia (GINTER e PIEPER, 2011). Contudo, a densidade da água é 800 vezes maior que a densidade do ar, dessa maneira, 12 milhas por hora (mph) a corrente marinha gera a quantidade equivalente de força que está contido em vento constante de 110 mph (KUSCHKE e STRUNZ, 2011), assim sendo, as turbinas hidrocinéticas são mais eficientes comparadas as turbinas eólicas. Além do mais, turbinas eólicas necessitam de uma grande área de terra de operação, enquanto turbinas hidrocinéticas operam sob água (KUSCHKE e STRUNZ, 2011; YUEN et al, 2009). 
O princípio de funcionamento da energia hidrelétrica das correntes de água pode ser visto na Figura 1, onde a energia cinética da corrente é convertida em potência mecânica, que roda um gerador para produzir eletricidade (HU e DU, 2012). A tecnologia da turbina hidrocinética ainda está em desenvolvimento e ainda não foi totalmente comercializado. Um fator, que joga um papel vital na comercialização, é a disponibilidade da turbina hidrocinética e sua energia: relação de custo (GUNEY e KAYGUSUZ, 2010)

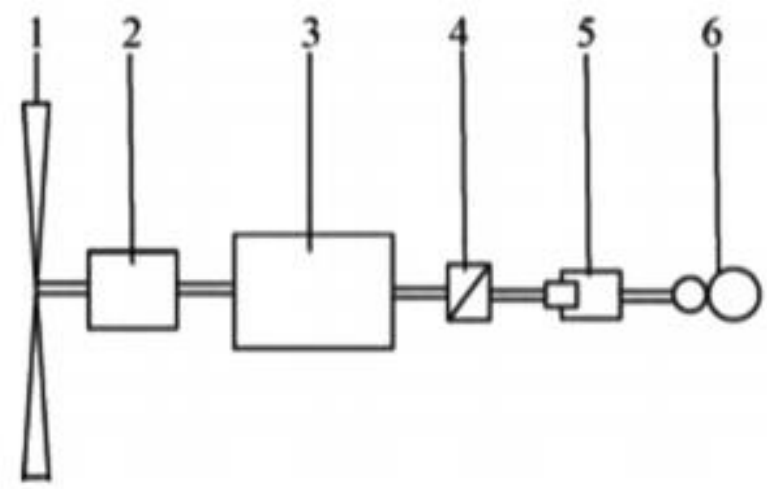

\section{Rotor 2.Gearbox 3.Generator 4.Converter/Inverter 5.Transformer 6.Grid connection}

Figura 1 - Esquema de turbina hidrocinética

Fonte: GUNEY e KAYGUSUZ (2010)

\section{- Rotor}

O rotor é o componente mecânico que compõe a turbina hidrocinética, composta por pás que são responsáveis por aproveitar a energia cinética presente no fluxo da água e transformá-las em energia cinética de rotação. A transmissão mecânica funciona como o acoplamento entre o eixo do rotor com o eixo do gerador (SANTOS, 2006). Os estudos nessa área são importantes para a otimização das formas e perfis de pás e para o avanço dessa tecnologia, pois podem aumentar a eficiência da turbina e torna o seu uso ainda mais interessante do ponto de vista da capacidade de fornecimento energético (FIGUEIREDO, 2013). Na Fig. 2, pode observar o fluxograma referente à classificação das turbinas hidrocinéticas. 


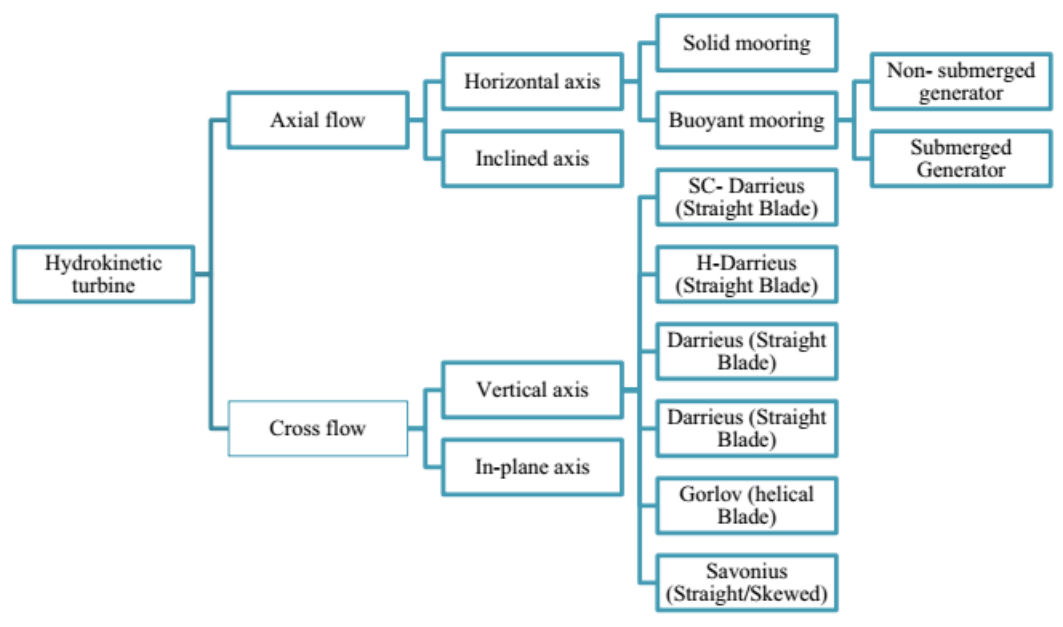

Figura 2 - Classificaçao de turbinas hidrocinéticas

Fonte: KHAN, IQBAL e QUAICOE (2008)

\section{- Rotores de eixo horizontal}

Rotores de eixo vertical, observadas na Fig. 3 são mais comumente utilizados, movidos por energias aerodinâmicas conhecidas como forças de sustentação (lift) que agem perpendicularmente ao escoamento e forças de arrasto (drag). Podem ser constituídos de apenas uma pá a até múltiplas pás, utilizando matérias como madeira alumínio ou fibra de vidro reforçada, em geral, na sua fabricação (CRESESB, 2017).

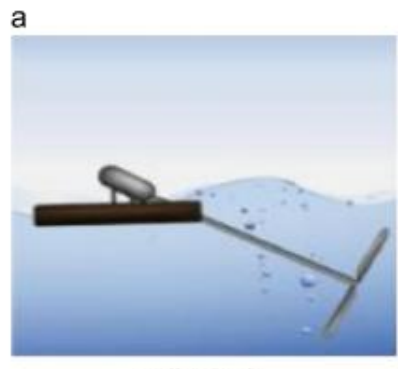

Inclined axis

C

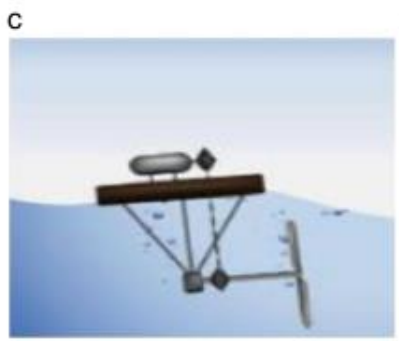

Non-submerged generator

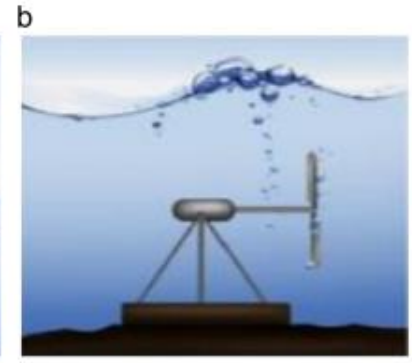

Rigid mooring

d

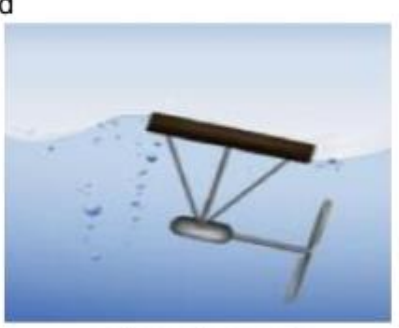

submerged generator

Figura 3 - Turbina de eixo horizontal

Fonte: KHAN, IQBAL e QUAICOE (2008)

Revista Brasileira de Energias Renováveis, v.8, n.4, p.653- 664, 2019 


\section{- Rotor de eixo vertical}

Rotores de eixo vertical, observadas na Fig. 4, têm a vantagem de não necessitarem de mecanismos de acompanhamento para variações da direção do vento, reduzindo a complexidade do projeto. Os rotores de eixo vertical também podem ser movidos por forças de sustentação (lift) e por forças de arrasto (drag). Os principais tipos de rotores de eixo vertical são Darrieus, Savonius e turbinas com torre de vórtices (LAYTON, 2010).

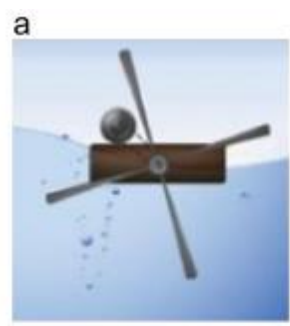

In-plane axis b

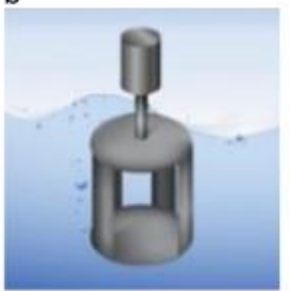

squirrel cage Darrieus

e

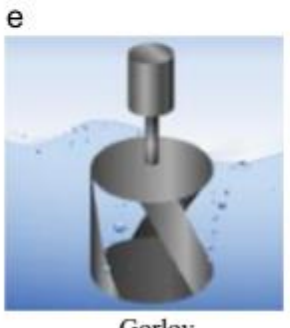

Gorlov
C

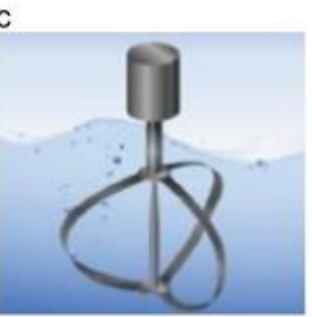

Darrieuse

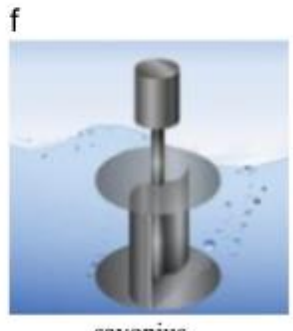

savonius

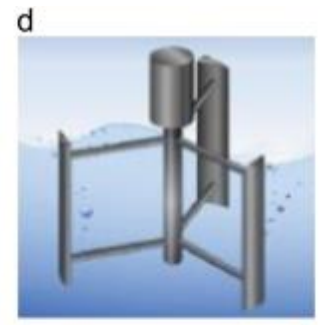

H-Darrieus

Figura 4 - Turbina de eixo vertical

Fonte: KHAN, IQBAL e QUAICOE (2008)

\section{- Transmissão e caixa multiplicadora}

A transmissão mecânica é o subsistema necessário para o acoplamento entre o eixo do rotor e o eixo do gerador. Para melhorar o rendimento da transmissão, devem ser observados parâmetros e conceitos sobre o assunto. A relação de transmissão, que é dada pela razão entre o diâmetro da polia movida pela polia motora, é um dos parâmetros que devem ser bem calculados, pois tem grande influência na geração do sistema (FIGUEIREDO, 2013).

\section{- Gerador}

O gerador de energia elétrica é o componente da usina, responsável pela conversão da energia oriunda da transmissão mecânica em energia elétrica. A viabilidade desse sistema 
depende do estudo das características dos rios, como velocidade da água, profundidade, perenidade e presença de entulhos (SILVA, 2011).

Atualmente a transformação de energia mecânica de rotação em energia elétrica através de equipamentos de conversão eletromecânica é um problema tecnologicamente já dominado, tanto é que existem várias alternativas de conjuntos moto-geradores, entre elas: geradores de corrente contínua, geradores síncronos, geradores assíncronos, geradores de comutador de corrente alternada. E cada um deles apresenta vantagens e desvantagens que devem ser analisadas com cuidado na incorporação em sistemas de conversão de energia (CRESESB, 2017).

\section{- Conversor}

Os sistemas de controle eletrônico da turbina têm a função de controlar a turbina, além de coletar os dados operacionais. Estes sistemas têm impacto significativo na produção de energia e estão se tornando cada vez mais avançados, controlando parâmetros como: Velocidade de rotação; temperaturas da hidráulica, inclinação do passo e da gola do nacelle de forma a verificar se estes são angulares à velocidade do vento (IRENA, 2012).

\section{- Transformador}

O tem a função regular a saída de tensão de rede. A tensão média de saída do gerador é intensificada pelo transformador para entre $10 \mathrm{kV} \mathrm{A} 35 \mathrm{kV}$; dependendo dos requisitos da rede local (IRENA, 2012).

\section{- Fatores de desempenho do SEBA}

O desempenho da HKT depende de vários parâmetros de projeto: Relação de aspecto (AR *), Relação de sobreposição, Rotor ângulo, ângulo de ataque ( $\alpha$ ), número de Reynolds $(\mathrm{Re})$, número de Froude, Relação de bloqueio (Br), Número de lâminas, Forma da lâmina, Solidez, Rotor colocação etc (LAGO, 2010; KAMOJI, 2009; ROY e SAHA, 2013). Além disso, a melhora do desempenho da HKT pode ser feita através da técnica de aumento multi-encenação, efeitos de superfície livres, objetos de camada limite a montante de turbinas, variação de largura de canal, área, aumento de duto e organizando turbina layout da disposição (KAILASH, ELDHO e PRABHU (2012), GROGAN et al, 2013; NEARY et al 2013).

Revista Brasileira de Energias Renováveis, v.8, n.4, p.653- 664, 2019 
Ao projetar um sistema HKT, são utilizados diferentes tipos de materiais para a fabricação da lâmina da turbina, para que seja garantida, alta proporção de força em peso, excelente resistência à fadiga e a maioria importante resistência à corrosão. Além disso, o uso de diversos tipos de materiais provou a capacidade retardadora na cavitação inícial mantendo o levantamento total e arraste (BEHROUZI et al, 2016). A maioria desses materiais de uso comum são reforçados com fibra de vidro Polímero (GFRP) para boas propriedades estruturais e baixo custo e Polímero reforçado com fibra de carbono (CFRP). Observa-se que CFRP de desempenho é melhor do que GFRP, mas é mais caro; assim sendo GFRP é principalmente preferido pelo fabricante (GINTER e PIEPER, 2011).

Observa-se dois métodos principais para melhorar a eficiência das turbinas e encontrar um alto valor de potência de saída que pode ser usado em Aplicações rio/oceâno: uma delas é aumentar a entrada Velocidade da corrente de água das turbinas usando duto ou bocal de fluxo, canais artificiais ou instalam-se turbinas após uma inclinação para aumentar a velocidade atual (BEHROUZI et al, 2016).

A confiabilidade da HKT depende da flutuação do torque, que, por sua vez, influenciam a qualidade do poder. Carga hidrodinâmica no gerador, isto é, a flutuação do torque deve ser minimizada em para produzir boa qualidade de energia, pois reduz a vibração da estrutura da turbina e da fadiga. Além disso, para melhorar o poder qualidade, caixa de velocidades moderna e gerador de energia elétrica podem ser incorporado, mas isso pode levar a aumentar o custo de geração (LI e ÇALISAL, 2010). Além do mais, os seguintes fatores têm impacto indireto no custo: desempenho do sistema e confiabilidade e aceitação social (Khan, Iqbal e Quaicoe, 2008). Confiabilidade está diretamente relacionada com a disponibilidade de produção de energia HKT e taxa de custo de energia. Geralmente, o modo de falha da turbina é fratura e fadiga devido ao carregamento final. Observa-se que fatores como U, o carregamento de HKT e a resistência do material afetam a confiabilidade da lâmina HKT (HU e DU, 2012).

Nas discussões acima, observa-se que a tecnologia, o desempenho de otimização de projeto, confiabilidade, análise técnico-econômica e o impacto ambiental desempenham um papel muito importante no design e implementação bem sucedida do sistema HKEC.

A viabilidade do projeto depende da análise e confiabilidade técnico-econômica. A verificação da qualidade da energia também é um fator importante ao projetar o sistema HKEC, que é derivado principalmente por análise de confiabilidade. Em caso de qualquer melhoria 
necessária em diferentes estágios de design da HKEC, são adotadas várias soluções e hipóteses. Durante a revisão da literatura, é observado que vários pesquisadores fizeram análises de valores em diferentes parâmetros de design da HKT. Percebe-se que entre as diferentes técnicas de aumento, há muita pesquisa alcance para explorar o efeito da carga hidráulica e do esforço induzido em HKT sob várias velocidades de fluxo livre em ângulo de rotor diferente com design de cortina e aumento de duto, o que pode melhorar o desempenho da HKT. Além disso, a análise deve ser feita para reduzindo o efeito indesejável como cavitação e erosão HKT usando a técnica de aumento (KUMAR et al., 2016).

\section{- Vantagens do sistema hidrocinético para obtenção de energia elétrica}

Permite a instalação em qualquer local que haja vento regular e satisfatório, mesmo em locais remotos, não requerendo instalações elétricas sofisticadas. Não há custo de consumo e sua manutenção se restringe à limpeza da bomba ou troca da bateria, quando utilizada, após longo período de uso (LIMA, 2009).

\section{- O uso do sistema hidrocinético para bombeamento de água}

A tecnologia de energia hidrocinética traz como benefícios a versatilidade, em decorrência de sua multiplicidade de dimensões e construções. Pode ser adequada as características do local de instalação, na procura da eficiência na transformação de energia disposta no leito. Dessa forma, verifica-se que a utilização de turbinas hidrocinéticas pode ser uma opção viável para comunidades isoladas próximas a rios que possuam características hidrológicas especifica (ELS; JUNIOR,2015). Onde a instalação destas turbinas podem ser aplicadas à sistemas de bombeamento de água com alta rapidez e eficiência, visto que os processos produtivos agrícolas demandam de grande volume de água para irrigação.

Tanto em aplicações na agricultura quanto na agropecuária, voltadas à irrigação, hidroponia, pulverização, abastecimento de bebedouros e reservatórios. Em indústrias e saneamento, no bombeamento, aeração e sistemas de recirculação de água. Em residências, condomínios e clubes no bombeamento de água proveniente de poços, lagos, rios e cisternas e na recirculação de piscinas (BARBUGENO e SILVA, 2003). 
O aproveitamento da energia gerada através de turbinas hidrocinéticas será de fundamental importância a curto prazo, devido ao baixo investimento e custos operacionais, as turbinas hidrocinéticas prometem ser rentáveis em comparação com outras tecnologias. O fornecimento contínuo de energia elétrica é uma grande vantagem em comparação com a energia solar ou eólica. Turbinas hidrocinéticas de pequena escala prometem ser uma solução para o fornecimento de energia em áreas remotas ou um substituto de baixo custo dos geradores a diesel. Devido ao custo relativamente baixo e a durabilidade dessas turbinas, os países em desenvolvimento podem produzir e implementar a tecnologia para fornecer a eletricidade necessária para pequenas comunidades (PIRES, 2014).

\section{Conclusões}

A aplicação de fontes renováveis de energia tem se mostrado de fundamental importância, principalmente quando voltada à pequenas propriedades rurais isoladas, visto que o acesso a fontes de energia renovável é um quesito decisório no desenvolvimento das mesmas.

A energia hidrocinética que por ser um sistema simplificado e de pouca manutenção, pode ser aplicado em pequenas propriedades rurais isoladas próximas de cursos hídricos, para o suprimento energético da mesma. Sendo que a instalação deve levar em consideração as características do local para a adequada seleção dos equipamentos adequados.

O Brasil, por apresentar um dos maiores potenciais hídricos do mundo, necessita de um aproveitamento de maneira mais eficiente possível desses recursos. Uma hipótese seria a utilização de turbinas hidrocinéticas para o bombeamento de água aplicado a atividades agrícolas. Para tal aproveitamento, se faz necessário investimentos para desenvolvimento e aplicação do sistema hidrocinético, além da disseminação das vantagens de sua utilização.

\section{Referências}

BEHROUZI, Fatemeh et al. Global renewable energy and its potential in Malaysia: A review of Hydrokinetic turbine technology. Renewable And Sustainable Energy Reviews, Malaya, v. 62, p.1270-1281, 2016.

CRESESB. Energia Eólica Princípios e Aplicações. Disponível em: http://paje.fe.usp.br/ mef pietro/mef2/app.upload/7/_mefmi_003-05.pdf 
EL BASSAM, N.; MAEGAARD, P. Integrated renewable energy for rural communities.

Amsterdam: Elsevier, 2005.

FIGUEIREDO, Sávio Weslley Oliveira et al. PROTÓTIPO HIDROCINÉTICO PARA GERAÇÃO DE ENERGIA ELÉTRICA APLICADO À EDUCAÇÃO EM ENGENHARIA. Revista de Ensino de Engenharia, Pará, v. 32, n. 1, p.19-30, 2013. Lago LI, Ponta FL, Chen L. Advances and trends in hydrokinetic turbine systems. Energy Sustain Dev 2010;14: 287-96.

Ginter VJ Pieper JK. Robust gain scheduled control of a hydrokinetic turbine. Control Syst Technol IEEE Trans 2011;19: 805-17.

Grogan DM, Leen SB, Kennedy CR. Brádaigh CMO. Design of composite tidal turbine blades. Renew Energy 2013; 57:151-62.

Guney MS, Kaygusuz K. Hydrokinetic energy conversion systems: a technology status review. Renew Sustain Energy Rev 2010;14: 2996-3004.

Hu Z, Du X. Reliability analysis for hydrokinetic turbine blades. Renew Energy 2012; 48:251-62

JUNIOR, A. C. P. B.; ELS, R. H. V. Turbina Hidrocinética geração 3. 2007.

Http://www2.aneel.gov.br/biblioteca/citenel2007/pdf/it46.pdf.

Kamoji MA, Kedare SB, Prabhu SV. Performance tests on helical Savonius rotors. Renew Energy 2009;34(3):521-9.

Kamoji MA, Kedare SB, Prabhu SB. Experimental investigations on single stage, two stages and three stage conventional Savonius rotor. Int J Energy Res 2008; 32:877-95.

Khan MJ, Bhuyan G, Iqbal MT, Quaicoe JE. Hydrokinetic energy conversion systems and assessment of horizontal and vertical axis turbines for river and tidal applications: a technology status review. Appl Energy 2009;86(10):1823-35.

Khan MJ, Iqbal MT, Quaicoe JE. River current energy conversion systems: progress, prospects and challenges. Renew Sustaina Energy Ver 2008; 12:2177-93

Kuschke M, Strunz K. Modeling of tidal energy conversion systems for smart grid operation. IEEE Power Energy Soc Gen Meeting 2011:1-3.

KUMAR, Dinesh; SARKAR, Shibayan. A review on the technology, performance, design optimization, reliability, techno-economics and environmental impacts of hydrokinetic energy

Revista Brasileira de Energias Renováveis, v.8, n.4, p.653- 664, 2019 
conversion systems. Renewable And Sustainable Energy Reviews, India, v. 58, p.796-813, 2016.

Lago LI, Ponta FL, Chen L. Advances and trends in hydrokinetic turbine systems. Energy Sustain Dev 2010;14: 287-96

Li Y, Çalisal SM. Numerical analysis of the characteristics of vertical axis tidal current turbines. Renew Energy 2010; 35:435-42.

Neary VS, Gunawan B, Hill C, Chamorro LP. Near and far field flow disturbances induced by model hydrokinetic turbine: ADV and ADP comparison. Renew Energy 2013; 60:1-6.

PIRES, L. B. M.. Geração de Energia Renovável através de Turbinas Hidrocinéticas. 2014.

Roy S, Saha UK. Review on the numerical investigations into the design and development of Savonius wind rotors. Renew Sustain Energy Rev 2013; 24:73- 83.

SANTOS, Alison A. D. et al. Projeto de Geração de Energia Eólica. Universidade Santa Cecilia. Santos. 2006.

SILVA, Teófilo R. M. D. Construção de um Protótipo Hidrocinético em Escala Reduzida para Geração de Energia Aplicado a Educação. Universidade Federal do Pará. Pará. 2011.

WU, Y. et al. Smart solar concentrators for building integrated photovoltaic façades. Solar Energy, v. 133, p. $111-118,2016$.

Yuen K, Thomas K, Grabbe M, Deglaire P, Bouquerel M, Österberg D. Matching a permanent magnet synchronous generator to a fixed pitch vertical axis turbine for marine current energy conversion. J IEEE 2009;2009 (34):24-31. 